\title{
MICAL-like Regulates Fasciclin II Membrane Cycling and Synaptic Development
}

\author{
Minyeop Nahm ${ }^{1,2}$, Sunyoung Park ${ }^{3}$, Jihye Lee ${ }^{4, *}$, and Seungbok Lee $e^{1,2,3, *}$
}

\begin{abstract}
Fasciclin II (FasII), the Drosophila ortholog of neural cell adhesion molecule (NCAM), plays a critical role in synaptic stabilization and plasticity. Although this molecule undergoes constitutive cycling at the synaptic membrane, how its membrane trafficking is regulated to ensure proper synaptic development remains poorly understood. In a genetic screen, we recovered a mutation in Drosophila mical-like that displays an increase in bouton numbers and a decrease in Fasll levels at the neuromuscular junction (NMJ). Similar phenotypes were induced by presynaptic, but not postsynaptic, knockdown of mical-like expression. Fasll trafficking assays revealed that the recycling of internalized Fasll molecules to the cell surface was significantly impaired in mical-like-knockdown cells. Importantly, this defect correlated with an enhancement of endosomal sorting of Fasll to the lysosomal degradation pathway. Similarly, synaptic vesicle exocytosis was also impaired in mical-like mutants. Together, our results identify Mical-like as a novel regulator of synaptic growth and Fasll endocytic recycling.
\end{abstract}

\section{INTRODUCTION}

Once initially formed during development, synapses undergo structural and functional modifications in response to genetic programs or environmental cues. This dynamic synapse feature is critical for the refinement of neural circuits during development and underlies learning and memory during animal life. Increasing evidence suggests that a family of cell adhesion molecules (CAMs), including vertebrate neural CAM (NCAM), Aplysia apCAM, and Drosophila Fasciclin II (Fasll), plays an important role in structural synaptic plasticity (Dalva et al., 2007; Packard et al., 2003). NCAM knockout mice display impair-

\footnotetext{
${ }^{1}$ Department of Brain and Cognitive Sciences, College of Natural Sciences, ${ }^{2}$ School of Dentistry and Dental Research Institute, ${ }^{3}$ Interdisciplinary Graduate Program in Brain Science, College of Natural Sciences, Seoul National University, Seoul 08826, Korea, ${ }^{4}$ Department of Oral Pathology and BK21 PLUS Project, School of Dentistry and Institute of Translational Dental Sciences, Pusan National University, Yangsan 50612, Korea *Correspondence: jihyelee@ @usan.ac.kr (JL); seunglee@snu.ac.kr (SL)
}

Received 24 August, 2016; revised 12 September, 2016; accepted 12 September, 2016; published online 21 October, 2016

Keywords: Drosophila, Fasll endocytic recycling, MICAL-like, neuromuscular junction, synaptic growth ments in hippocampal long-term potentiation (LTP) and spatial memory (Bukalo et al., 2004; Cremer et al., 1994; Muller et al., 1996). In Aplysia sensorimotor co-cultures, serotonin-induced long-term facilitation (LTF) is accompanied by the formation of new synaptic connections between sensory and motor neurons (Glanzman et al., 1990). This structural change is associated with downregulation of apCAM surface expression in presynaptic sensory neurons (Mayford et al., 1992). Similarly, at the Drosophila neuromuscular junction (NMJ), mutations that increase neuronal activity (e.g., eag Shaker) and cAMP levels (e.g., dunce) cause a decrease in synaptic Fasll and a concurrent increase in bouton number (Schuster et al., 1996b). Consistently, hypomorphic fas/l mutations that decrease Fasll levels also cause synaptic overgrowth (Schuster et al., 1996a). Importantly, synaptic overgrowth in eag Shaker and dunce is suppressed by transgenic expression to maintain synaptic Fasll levels (Schuster et al., 1996b), supporting the role of Fasll downregulation in activity-dependent synaptic growth. Collectively, these findings suggest that synaptic growth is negatively regulated by synaptic levels of NCAM-type proteins.

Endocytosis and subsequent lysosomal degradation of NCAM-type proteins are apparently the key mechanisms that reduce cell adhesion during synaptic growth. In Aplysia sensory neurons, serotonin downregulates synaptic apCAM by promoting its internalization and rerouting from a recycling to a lysosomal degradation pathway (Bailey et al., 1992; Mayford et al., 1992). Subsequent experiments suggested that the internalization of apCAM requires mitogen-activated protein kinase (MAPK)-dependent phosphorylation in its PEST sequence (Bailey et al., 1997; Michael et al., 1998). MAPK signaling has also been implicated in activity-dependent downregulation of synaptic Fasll at the Drosophila NMJ. Increased neural activity in some paralytic mutants causes persistent activation of neuronal MAPK signaling (Hoeffer et al., 2003), which in turn leads to reduced synaptic Fasll and increased synaptic growth (Hoeffer et al., 2003; Koh et al., 2002). Taken together, these findings indicate the important role of endocytic downregulation of NCAM-type proteins in synaptic plasticity. However, knowledge regarding the molecular machinery controlling endocytosis and endosomal trafficking of NCAM-type proteins is limited.

In mammalian cells, efficient recycling of endocytosed receptors and CAMs to the plasma membrane is mediated by two members of the molecules interacting with CasL (Mical) protein family, Mical-like 1 (Mical-L1) and Mical-like 2 (Mical-L2), which share an N-terminal calponin homology $(\mathrm{CH})$ domain, a central Lin11, Isl-1, and Mec-3 (LIM) domain, and a C-terminal coiledcoil (CC) domain that can interact with several Rab-family pro- 
teins (Fukuda et al., 2008; Yamamura et al., 2008). Mical-L1 has been proposed to facilitate endocytic recycling of transferrin and $\beta 1$ integrin receptors by recruiting and linking the endocytic regulators Rab8a and EH domain-containing protein 1 (EHD1) on recycling endosomes (Sharma et al., 2009). In addition, Mical-L2 is shown to interact with Rab8 and Rab13 to facilitate endocytic recycling of E-cadherin and occudin, respectively, in epithelial cells (Terai et al., 2006; Yamamura et al., 2008). Although the interaction of Mical-L2 with Rab13 is essential for neurite outgrowth in cultured PC12 cells (Sakai et al., 2010), its role in the nervous system remains poorly understood.

A single Mical-like ortholog is expressed in Drosophila (Giridharan and Caplan, 2014). In this study, we examine the synaptic function of Mical-like at the Drosophila NMJ. We show that Mical-like acts presynaptically to restrain synaptic growth and maintain normal levels of synaptic Fasll. In addition, loss of Mical-like impairs the recycling of internalized Fasll molecules to the cell surface and enhances their degradative endosomal trafficking. Our results reveal a novel role for Mical-like in the regulation of Fasll membrane trafficking and synaptic development.

\section{MATERIALS AND METHODS}

\section{Drosophila strains}

All fly strains were reared on standard Drosophila food at $25^{\circ} \mathrm{C}$. $w^{1118}$ was used as the wild-type strain. A P-element allele of mical-like (G8603) was obtained from GenExel (Korea). A transgenic line carrying UAS-mical-like ${ }^{R N A i}$ was obtained from the Bloomington Stock Center (USA). Expression of this transgene was driven by either the neuron-specific C155-GAL4 driver (Lin and Goodman, 1994) or the muscle-specific BG57GAL4 driver (Budnik et al., 1996).

\section{Molecular biology}

To generate $p A c-M y c$-fasll, Drosophila fas/l cDNA was amplified by PCR from genomic DNA prepared from a UAS-FasllYFP transgene (Kohsaka et al., 2007) and then subcloned into the pTOP Blunt V2 vector (Enzynomics, Korea). A c-Myc epitope sequence was introduced immediately downstream of the signal sequence of fas/l via PCR-based mutagenesis. The resulting c-Myc-fas/l insert was subcloned into the pAc5.1 vector (Invitrogen, USA) for expression in Drosophila S2R+ cells.

\section{Cell culture and transfection}

$\mathrm{S} 2 \mathrm{R}+$ cells were cultured at $25^{\circ} \mathrm{C}$ in Schneider's medium (Invitrogen, USA) supplemented with $10 \%$ heat-inactivated fetal bovine serum (FBS). The cells were transfected in six-well plates using Cellfectin (Invitrogen, USA). In each experiment, 1 $\times 10^{6}$ cells were transfected with $2 \mu \mathrm{g}$ of plasmid DNA or $5 \mu \mathrm{g}$ of dsRNA in six well plates.

For RNAi-mediated depletion of Mical-like in S2R+ cells, mical-like dsRNA was produced by in vitro transcription of a DNA template containing T7 promoter sequences at both ends, as previously described (Nahm et al., 2010b). The DNA template for in vitro transcription was generated by PCR using primers containing a T7 promoter sequence upstream of the following mical-like-specific sequences: 5 '-TTGGAGTATTGGTGCCGAGTT-3' and 5'-CTCCTCTGGCTTCGGGTGCTT-3'.

Immunohistochemistry and morphological quantification of larval NMJs

Wandering third instar larvae were collected and dissected in ice-cold $\mathrm{Ca}^{2+}$-free $\mathrm{HL} 3$ saline. Larval fillets were fixed for $20 \mathrm{~min}$ in $4 \%$ formaldehyde in phosphate-buffered saline (PBS). Antibody staining for fixed samples was performed as previously described (Nahm et al., 2010a). The following primary antibodies were used in this study: mouse anti-Fasll (1D4; DSHB, USA) at 1:20 and FITC-conjugated goat anti-HRP (Jackson ImmunoResearch Laboratories, USA) at 1:200. A Cy3conjugated donkey anti-mouse secondary antibody (Jackson ImmunoResearch Laboratories, USA) was used at a dilution of 1:200. Confocal images were taken with an Olympus FV300 laser-scanning confocal microscope using a Plan Apo 40×0.90 NA or U Plan Apo 100x 1.35 NA objective and processed using FLOUVIEW imaging analysis software. To compare different genotypes, samples for each experiment were prepared simultaneously and imaged under identical confocal settings.

Bouton number was quantified at NMJ $6 / 7$ in abdominal segment 2 after anti-HRP staining as previously described (Nahm et al., 2010a). For quantification of synaptic Fasll levels, larval NMJs were double-stained with anti-HRP and anti-Fasll antibodies. The fluorescence intensity of synaptic Fasll was measured using ZEN imaging software (Carl Zeiss, Germany) and then normalized to HRP intensity.

Cell surface FasIl expression assay

$\mathrm{S} 2 \mathrm{R}+$ cells transfected with the $p A c-M y c$-fas/l construct with or without mical-like dsRNA were incubated for $72 \mathrm{~h}$ and then treated with $100 \mu \mathrm{g} / \mathrm{ml}$ cycloheximide for $5 \mathrm{~h}$ to inhibit protein synthesis. To visualize surface Fasll molecules, the cells were incubated with rabbit anti-Myc antibody (Cell Signaling, USA; $1: 100$ ) in culture medium for $1 \mathrm{~h}$ at $4^{\circ} \mathrm{C}$ and then fixed for 20 min with $4 \%$ formaldehyde in PBS. After washing three times with PBS, cells were incubated with Cy3-conjugated donkey anti-rabbit secondary antibody (Jackson ImmunoResearch Laboratories, USA; 1:200) for $30 \mathrm{~min}$. To visualize the total pool of Fasll, the same cells were then permeabilized with $0.2 \%$ Triton X-100 in PBS for 10 min and sequentially incubated with mouse anti-Fasll antibody (DSHB, USA; 1:50) for $1 \mathrm{~h}$ and FITC-conjugated donkey anti-mouse secondary antibody (Jackson ImmunoResearch Laboratories, USA; 1:200) for 30 min. S2R+ cells showed no detectable levels of endogenous Fasll based on anti-Fasll immunostaining. Images of immunostained cells were acquired using a LSM 700 laserscanning confocal microscope (Carl Zeiss, Germany). Images were processed using the ZEN imaging software. To quantify surface Fasll expression, the fluorescence intensity of surface Myc-Fasll (red) was normalized to the total Myc-Fasll (green) fluorescence intensity.

\section{Fasll trafficking assay}

For the Fasll recycling assay, live S2R+ cells transfected with pAc-Myc-fas/l were incubated with rabbit anti-Myc antibody $(1: 100)$ in culture medium at $4^{\circ} \mathrm{C}$ for $1 \mathrm{~h}$ to label surface MycFasll proteins. Cells were incubated in culture medium at $25^{\circ} \mathrm{C}$ for $30 \mathrm{~min}$ to allow internalization and then washed with ice-cold acid buffer (0.5 M NaCl, $0.2 \mathrm{M}$ acetic acid, $\mathrm{pH} 4.5)$ for 4 min to remove the antibodies remaining on the cell surface. After washing three times with culture medium, cells were incubated in culture medium at $25^{\circ} \mathrm{C}$ for $1 \mathrm{~h}$ to allow recycling of the internalized Fasll-antibody complex to the cell surface. Following fixation, cells were incubated with Cy3-conjugated anti-rabbit secondary for $30 \mathrm{~min}$ to visualize the surface Fasll pool. Subsequently, cells were permeabilized with $0.2 \%$ Triton X-100 in PBS for $10 \mathrm{~min}$ and stained with FITC-conjugated anti-rabbit secondary antibody for 30 min to visualize the internalized Fasll 
A

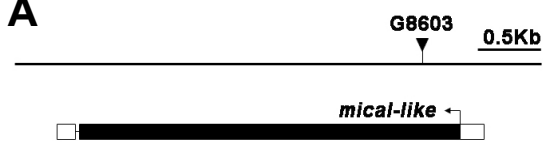

B

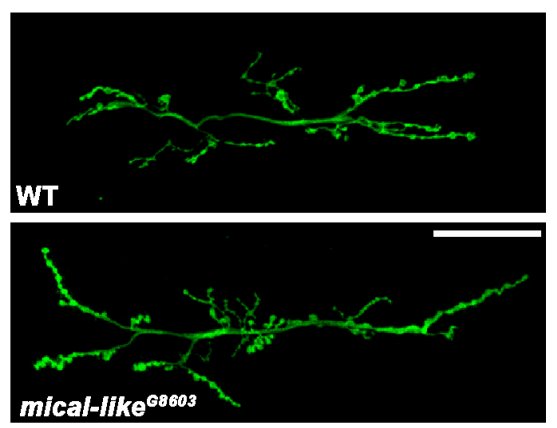

C

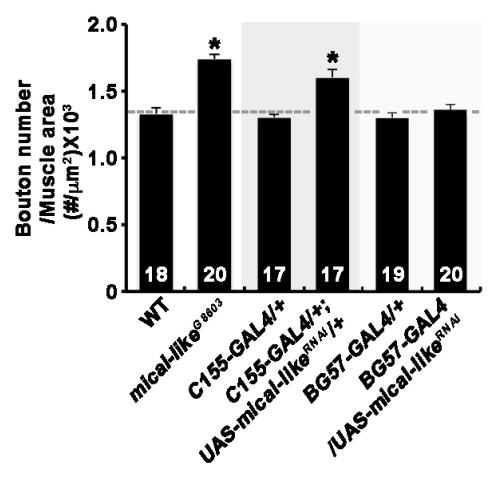

Fig. 1. Loss of presynaptic Mical-like results in synaptic overgrowth at the NMJ. (A) Genomic organization of the mical-like locus showing the position of the P-element insertion G8603 (inverted triangle). Introns and exons are indicated by horizontal lines and boxes, respectively. Untranslated regions are indicated by white boxes, the translated region by a black box, and the translation initiation site by an arrow. (B) Confocal images of anti-HRP-stained NMJs $6 / 7$ of larval abdominal segment 2 in wild-type and mical-like ${ }^{68603}$ third instar larvae. (C) Quantification of total bouton number normalized to muscle surface area at NMJ 6/7 in third instar larvae of the indicated genotypes. The number of analyzed NMJs is indicated within each bar. Data are presented as means \pm SEM. Statistically significant differences versus wild-type or the corresponding GAL4 control are indicated $\left({ }^{*} P<0.001\right)$. Scale bar, $50 \mu \mathrm{m}$. pool. For quantification of Fasll recycling, the fluorescence intensity of recycled Myc-Fasll (red) was normalized to internal Myc-Fasll (green) fluorescence intensity.

To analyze endosomal sorting of internalized Fasll proteins to lysosomes, S2R+cells transfected with $p A C-M y c$-fasll and pAc-GFP-rab7 or pAc-spin-GFP were surface-labeled with rabbit anti-Myc antibody as described above, washed, and then incubated in culture medium for $30 \mathrm{~min}$ at $25^{\circ} \mathrm{C}$ to allow internalization. Following fixation and permeabilization, cells were incubated with mouse anti-GFP antibody (Merck Millipore, Germany; $1: 100$ ) for $1 \mathrm{~h}$ and sequentially with Cy3-conjugated donkey anti-rabbit and FITC-labeled donkey anti-mouse secondary antibodies for $30 \mathrm{~min}$.

\section{FM1-43 dye experiments}

FM1-43 dye loading and unloading at the larval NMJ were achieved as previously described (Verstreken et al., 2008). Briefly, wandering third-instar larvae were dissected in $\mathrm{Ca}^{2+}$ free HL3 saline and incubated in HL3 saline containing $90 \mathrm{mM}$ $\mathrm{KCl}, 5 \mathrm{mM} \mathrm{CaCl}$, and $4 \mu \mathrm{M} \mathrm{FM1-43} \mathrm{(Molecular} \mathrm{Probes,} \mathrm{USA)}$ for $1 \mathrm{~min}$. Excessive dye was washed away with $\mathrm{Ca}^{2+}$-free HL3 saline before imaging of loaded NMJ synapses. Subsequently, NMJ synapses were subjected to a second stimulation with 90 $\mathrm{mM} \mathrm{K}^{+}$saline for 15 or 30 min, washed with $\mathrm{Ca}^{2+}$-free HL3 saline, and imaged for the remaining dye. Images of NMJ synapses were acquired using a Plan-NEOFLUAR 40×0.75 NA water-immersion lens on the Axio Imager D1 epifluorescence microscope (Karl Zeiss, Germany), and the fluorescence intensity was quantified using $\mathrm{NIH}$ ImageJ.

\section{RESULTS}

Mical-like is required presynaptically to restrain synaptic growth at the Drosophila larval NMJ

In a genetic screen for mutations that affect synaptic morphology at the Drosophila larval NMJ (Nahm et al., 2010a; 2010b), we identified an enhancer $P$ (EP) insertion, G8603, that resides in the first exon of the mical-like gene (Fig. 1A). mical-like ${ }^{G 8603}$ mutants were homozygous viable and had more extensive NMJs than wild-type ( $w^{1118}$ ) controls (Fig. 1B). To quantify the mical-like phenotype, we counted the number of synaptic bou- tons on muscles 6 and 7 of abdominal segment $A 2$ of thirdinstar larvae. Because NMJ growth is coupled with an increase in muscle size (Schuster et al., 1996a), we normalized bouton numbers to muscle surface area. Normalized bouton number was increased by $30 \%$ in mical-like mutants compared with wild-type larvae $\left(1.74 \pm 0.03\right.$ versus $1.33 \pm 0.04 \times 10^{3}$ boutons/ $\mathrm{mm}^{2} ; P<0.001$; Fig. $\left.1 \mathrm{C}\right)$.

To determine whether Mical-like is required pre- or postsynaptically, we knocked down mical-like in a tissue-specific fashion using a transgenic RNA interference (RNAi) approach. When UAS-mical-like ${ }^{R N A i}$ was driven by a pan-neuronal C155GAL4 driver, we observed a $23 \%$ increase in the number of boutons (Fig. 1C). In contrast, expression of UAS-mical-like RNAi with the muscle-specific BG57-GAL4 driver had no significant effect on overall bouton number (Fig. 1C). These data indicate that Mical-like function is required presynaptically for normal synaptic growth at the NMJ.

\section{Mical-like is essential for the normal abundance of Fasll at} the NMJ

Given the role of Mical-like in the regulation of synaptic growth, we further examined mical-like third instar NMJs with several synaptic markers. The levels and distribution of the presynaptic vesicle marker Cysteine-string protein (Csp), the presynaptic active zone marker Bruchpilot/NC82, and the postsynaptic subsynaptic reticulum (SSR) marker Discs-large (Dlg) were grossly normal in homozygous mical-like ${ }^{G 603}$ mutants (data not shown). However, we observed a $40 \%$ reduction in the level of the cell adhesion molecule Fasll $(P<0.001$; Figs. $2 \mathrm{~A}$ and $2 \mathrm{~B})$. A similar decrease of synaptic Fasll levels was also induced by presynaptic knockdown of mical-like based on RNAi (Fig. 2B), consistent with its effect on synaptic growth (Fig. 1C). In contrast, a change in Fasll levels following knockdown of mical-like in postsynaptic muscles could not be detected (Fig. 2B). These data reveal a presynaptic role of Mical-like in the regulation of Fasll levels.

Mical-like is required for the recycling of internalized Fasll proteins to the cell surface

We investigated how Mical-like downregulates synaptic Fasll. Mammalian Mical-like homologs (Mical-L1 and Mical-L2) have 
A

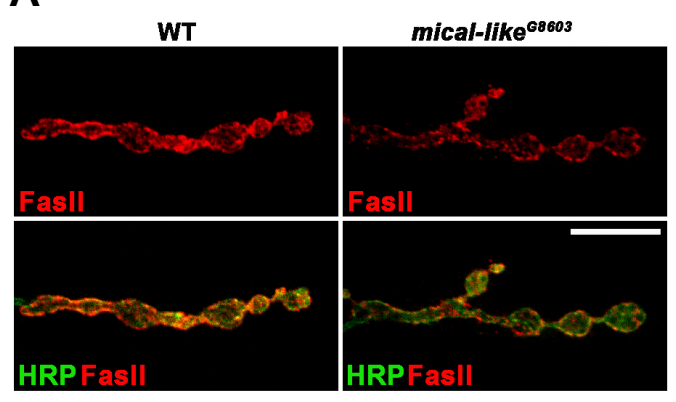

B

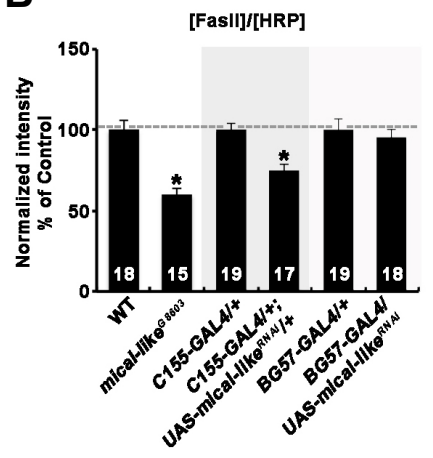

Fig. 2. Loss of presynaptic Mical-like decreases levels of synaptic Fasll. (A) Confocal images of wild-type and mical-like ${ }^{68603}$ third instar NMJs 6/7 stained with anti-Fasll (red) and anti-HRP (green). (B) Quantitative measurements of anti-Fasll fluorescence intensity relative to anti-HRP intensity at NMJ $6 / 7$ in third instar larvae of the indicated genotypes. The number of analyzed NMJs is indicated within each bar. Data are presented as means \pm SEM. Statistically significant differences versus wild-type or the corresponding GAL4 control are indicated ( $\left.{ }^{\star} P<0.001\right)$. Scale bar, $10 \mu \mathrm{m}$.
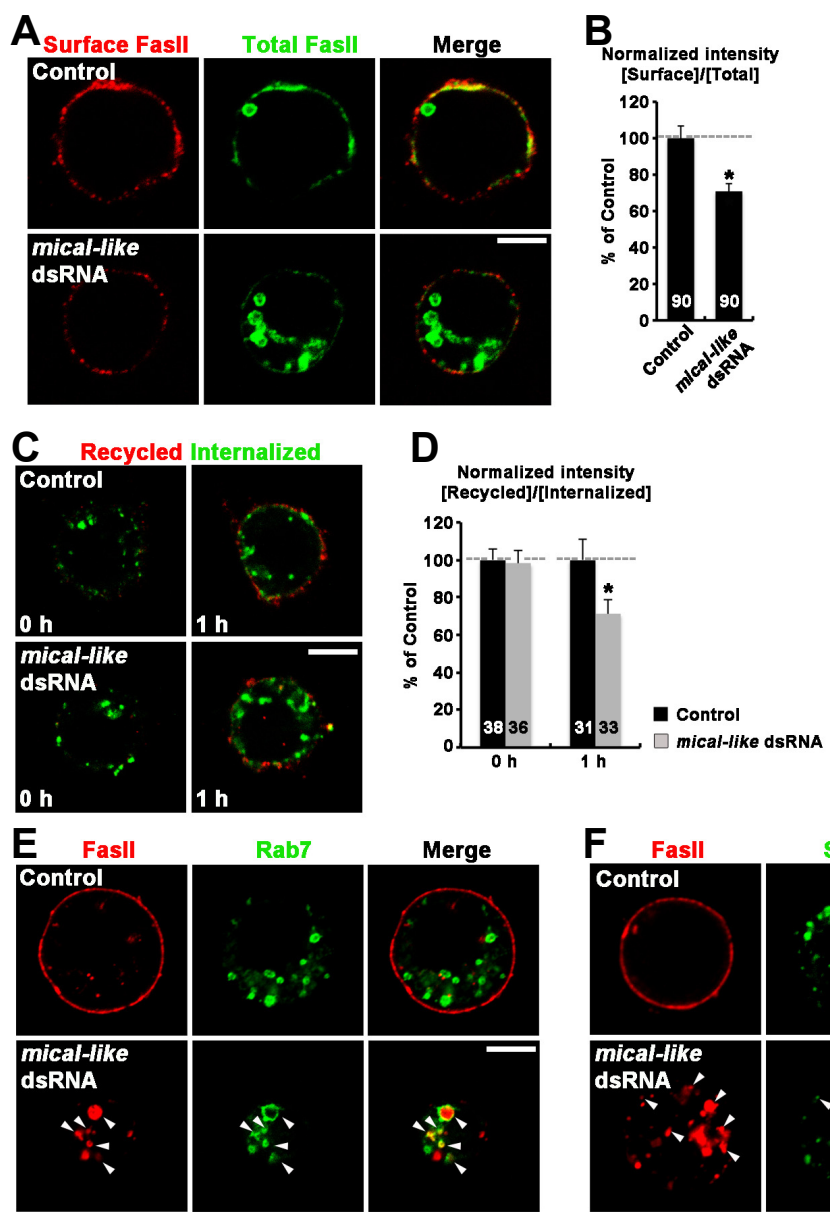

anti-Myc and anti-GFP antibodies. Arrowheads indicate GFP-Rab7
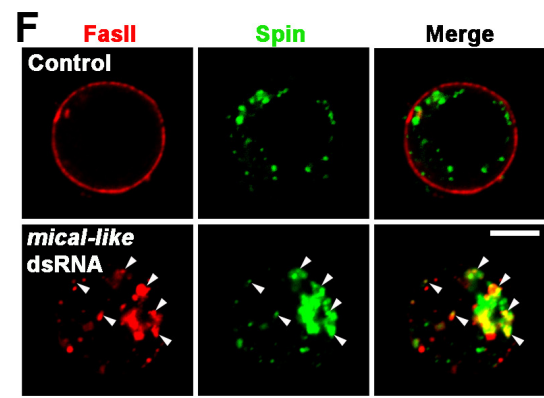

Fig. 3. Loss of Mical-like impairs the recycling of internalized Fasll molecules to the cell surface. (A, B) Mical-like-dependent regulation of surface Fasll expression. (A) $\mathrm{S} 2 \mathrm{R}+$ cells transfected with $p A c-M y c-f a s / l$ in the absence (control) or presence of micallike dsRNA were treated with cycloheximide for $5 \mathrm{~h}$ to block new protein synthesis, fixed, and sequentially stained for the surface (red) and total (green) Myc-Fasll pools under nonpermeant and permeant conditions, respectively. (B) Quantification of surface-to-total Myc-Fasll ratio. (C, D) Mical-like is required for efficient recycling of endocytosed Fasll proteins to the cell surface. (C) S2R+ cells expressing Myc-Fasll were incubated live with anti-Myc antibody at $4^{\circ} \mathrm{C}$ and then subjected to Fasll recycling assays (see "Materials and Methods"). Recycled and internalized Myc-Fasll proteins were visualized by staining with Cy3and FITC-conjugated anti-rabbit secondary antibody under nonpermeant and permeant conditions, respectively. (D) Quantification of recycled-to-internalized Myc-Fasll ratio. $(E, F)$ Myc-Fasll proteins internalized in the absence of Mical-like accumulate in late endosomes/lysosomes. S2R+ cells transfected with $p A c-M y c$-fas $/ l$ and $p A c-G F P$ rab7 (E) or pAc-Spin-GFP (F) in the absence (control) or presence of mical-like dsRNA were surface-labeled with anti-Myc antibody and incubated at $25^{\circ} \mathrm{C}$ for $30 \mathrm{~min}$ to allow endocytosis. After fixation and permeabilization, cells were stained with both nalized Myc-Fasll proteins (red). ${ }^{*} P<0.001$. Scale bars, $5 \mu \mathrm{m}$.

been implicated in the regulation of endocytic recycling of junctional proteins in non-neuronal cells (Sharma et al., 2009; Terai et al., 2006; Yamamura et al., 2008). In addition, Fasll is shown to cycle between the plasma membrane and intracellular endocytic compartments at the Drosophila NMJ (Mathew et al., 2003). Based on these findings, we hypothesized that Mical-like plays a role in synaptic Fasll cycling. To test this possibility, we transiently transfected a Myc-Fasll construct into Drosophila S2 cells. The surface and total pools of Fasll were visualized by immunostaining with anti-Myc antibody before cellular permeabilization and subsequently with anti-Fasll antibody following permeabilization, respectively. The ratio of surface to total MycFasll levels was decreased by approximately $29 \%$ in cells treated with mical-like dsRNA compared with mock-treated control cells (Figs. $3 \mathrm{~A}$ and $3 \mathrm{~B}$ ). This change in Fasll surface expression supports the role of Mical-like in the endocytic 
A

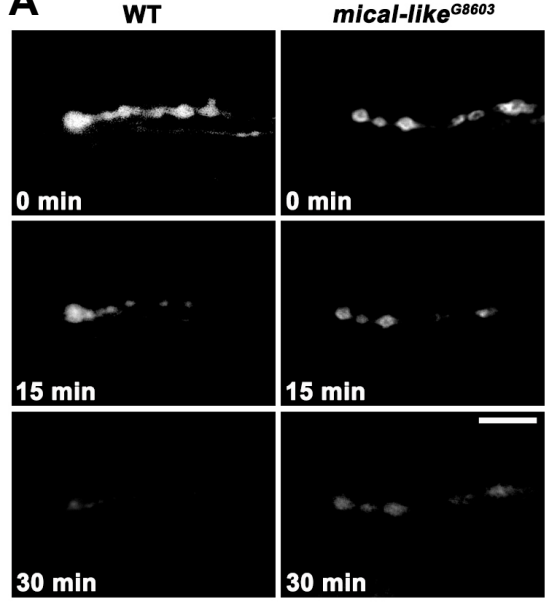

B

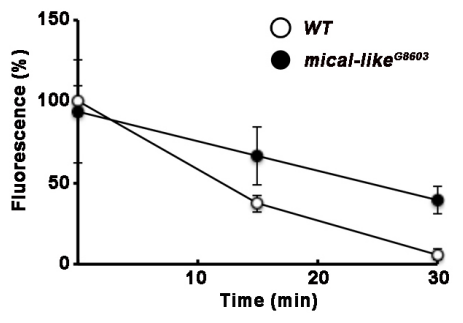

Fig. 4. FM1-43 dye imaging shows a significant defect in SV exocytosis in mical-like mutants. (A) Images of wild-type and mical-like ${ }^{G 8603}$ third instar NMJs 6/7 after dye loading and unloading. NMJ preparations were stimulated with saline containing $90 \mathrm{mM} \mathrm{K}^{+}$for $1 \mathrm{~min}$ in the presence of FM143 to load newly endocytosed SVs, washed with $\mathrm{Ca}^{2+}$-free saline to stop SV cycling, and then imaged to assess dye loading (0 $\mathrm{min})$. The same NMJ preparations were re-stimulated using saline containing $90 \mathrm{mM} \mathrm{K}^{+}$for 15 or $30 \mathrm{~min}$ in the presence of FM1-43, washed with $\mathrm{Ca}^{2+}$-free saline, and then imaged to assess dye unloading via SV exocytosis. Scale bar, $10 \mu \mathrm{m}$. (B) Quantification of mean fluorescence intensity in synaptic boutons after FM1-43 loading and unloading. Values are presented as percentages of wild-type boutons after dye loading. downregulation of Fasll.

Next, we directly investigated the role of Mical-like in the recycling of internalized Fasll to the plasma membrane. Live $\mathrm{S} 2 \mathrm{R}+$ cells expressing Myc-Fasll were surface-labeled with anti-Myc antibody at $4^{\circ} \mathrm{C}$, a condition that does not permit endocytosis. Cells were then incubated at $25^{\circ} \mathrm{C}$ for $30 \mathrm{~min}$ to allow internalization of the Myc-Fasll-antibody complexes. After stripping away non-internalized antibodies using an acid buffer, the cells were further incubated at $25^{\circ} \mathrm{C}$ for $1 \mathrm{~h}$ to allow recycling of the internalized Myc-Fasll-antibody complex back to the plasma membrane. Surface and intracellular Myc-Fasll protein levels were determined by anti-Myc immunostaining under nonpermeant and permeant conditions, respectively, after the acid wash or additional $1 \mathrm{~h}$ incubation. After the acid wash, there was no significant difference in the amount of internalized Myc-Fasll-antibody complexes between mical-like-knockdown and mock-treated control cells (Figs. 3C and 3D). In contrast, the level of surface-recycled Myc-Fasll-antibody complexes at $1 \mathrm{~h}$ after the acid wash was significantly reduced in mical-likeknockdown cells compared with mock-treated controls (Figs. $3 \mathrm{C}$ and $3 \mathrm{D}$ ). These results suggest that Mical-like is required for endocytic recycling but not internalization of Fasll proteins.

Finally, we monitored endosomal trafficking of the internalized Fasll in comparison with the late endosomal/lysosomal markers Rab7 and Spinster (Spin). To do so, live control and mical-like knockdown S2R+ cells expressing Myc-Fasll and GFP-Rab7 or Spin-GFP were prelabeled with anti-Myc antibody at $4^{\circ} \mathrm{C}$ and then incubated at $25^{\circ} \mathrm{C}$ for $30 \mathrm{~min}$ to resume endocytosis prior to immunostaining for Myc and GFP under permeant conditions. In control cells, the internalized Myc-Fasll poorly overlapped with GFP-Rab7 and Spin-GFP (Figs. 3E and 3F). In contrast, we observed extensive overlap between Myc-Fasll and the late endosomal/lysosomal markers in mical-like-knockdown cells (Figs. 3E and 3F). These results indicate that the impaired recycling of Fasll in the absence of Mical-like is coupled with its preferential sorting to the degradative compartment.

Altogether, these data imply that Mical-like maintains synaptic Fasll levels by promoting the reincorporation of internalized Fasll to the synaptic membrane.

Mical-like is required for synaptic vesicle exocytosis at the NMJ

The lipophilic dye FM1-43 can reversibly bind to membranes and has been used to monitor synaptic vesicle (SV) endocytosis and exocytosis at the Drosophila larval NMJ (Verstreken et al., 2008). To examine whether Mical-like also plays a role in exocytosis of SVs in addition to Fasll, we monitored the loading and unloading kinetics of FM1-43 at mical-like ${ }^{G 8603}$ synapses. Laval NMJ arbors were first loaded with FM dye by depolarizing NMJ synapses using saline containing $90 \mathrm{mM} \mathrm{K}^{+}$, and then washed with $\mathrm{Ca}^{2+}$-free saline to stop SV cycling. Subsequently, FM dye was unloaded by applying a second depolarizing stimulus. Under these conditions, dye loading was not significantly different between mical-like ${ }^{G 8603}$ and wild-type animals (Fig. 4A) Subsequently, wild-type boutons were almost completely unloaded by the second depolarizing stimulus within 30 min (Figs. $4 \mathrm{~A}$ and $4 \mathrm{~B}$ ). However, at this time point, mical-like ${ }^{G 8603}$ boutons showed a high retention of FM1-43 (Figs. 4A and 4B). Taken together, these data indicate that mical-like mutants are severely defective in SV exocytosis, but not endocytosis.

\section{DISCUSSION}

Mammalian Mical-like proteins Mical-L1 and Mical-L2 play a role in endocytic recycling of cell surface receptors or junctional proteins in non-neuronal cells (Sharma et al., 2009; Terai et al., 2006; Yamamura et al., 2008). Although these proteins are expressed in neurons (Sakai et al., 2010), their neuronal function remains unclear. Here, in a genetic screen for mutations causing defects in NMJ development, we identify mical-like encoding a single Drosophila ortholog of Mical-like proteins. mical-like mutant NMJs show an increase in bouton number and a decrease in Fasll levels. Our targeted RNAi knockdown experiments demonstrate that Mical-like acts presynaptically to control synaptic growth and Fasll levels.

Is there a relationship between two synaptic phenotypes induced by loss of Mical-like? Prior studies on the NMJ have demonstrated two main phenotypes of various Fasll mutants depending on Fasll levels (Schuster et al., 1996a). In Fasll null mutants, synaptic boutons form normally during embryogenesis but subsequently retract during larval development. In contrast, hypomorphic fasll mutants, which express Fasll above a threshold for supporting synaptic maintenance, show synaptic overgrowth. These findings suggest that Fasll-mediated cell adhesion is essential for synaptic maintenance, but also constrains further synaptic expansion when Fasll expression is 
beyond a certain level. Thus, it is tempting to speculate that Mical-like restrains synaptic growth by maintaining synaptic Fasll levels.

At the Drosophila NMJ, Fasll is shown to constitutively cycle between the plasma membrane and intracellular compartments (Mathew et al., 2003), suggesting that Fasll-mediated cell adhesion could be regulated by endocytic membrane trafficking. Despite this emerging outline for synaptic Fasll membrane cycling, the identity of the molecular machinery governing this process is poorly defined. At the postsynaptic terminal, the reincorporation of internalized Fasll molecules to the cell surface is accomplished by an amphiphysin/soluble N-ethylmaleimidesensitive factor attachment protein receptor (SNARE)-dependent mechanism (Mathew et al., 2003; Packard et al., 2003). Our current data uncover a novel mechanism of presynaptic Fasll recycling, which is mediated by Mical-like. Loss of presynaptic, but not postsynaptic, Mical-like decreases Fasll levels at NMJ synapses. We also show that loss of Mical-like selectively impairs the recycling of internalized Fasll molecules to the cell surface without affecting endocytosis. Finally, our FM1-43 dye uptake/release experiments also reveal the selective role of Mical-like in SV exocytosis at the NMJ.

In conclusion, our data demonstrate a conserved role for Drosophila Mical-like in regulating endocytic recycling and indicate that this role of Mical-like is important for the proper regulation of Fasll levels at synapses. Given the importance of local Fasll regulation for structural synaptic plasticity, it will be interesting to determine in the future whether the activity of Micallike to promote Fasll recycling is finely regulated in an activitydependent manner.

\section{ACKNOWLEDGEMENTS}

This study was supported by grants from the National Research Foundation (grant numbers 2010-0029398 and 20110019228 to S.L.).

\section{REFERENCES}

Bailey, C.H., Chen, M., Keller, F., and Kandel, E.R. (1992). Serotonin-mediated endocytosis of apCAM: an early step of learning-related synaptic growth in Aplysia. Science 256, 645649.

Bailey, C.H., Kaang, B.K., Chen, M., Martin, K.C., Lim, C.S., Casadio, A., and Kandel, E.R. (1997). Mutation in the phosphorylation sites of MAP kinase blocks learning-related internalization of apCAM in Aplysia sensory neurons. Neuron 18, 913-924.

Budnik, V., Koh, Y.H., Guan, B., Hartmann, B., Hough, C., Woods, D., and Gorczyca, M. (1996). Regulation of synapse structure and function by the Drosophila tumor suppressor gene dlg. Neuron 17, 627-640.

Bukalo, O., Fentrop, N., Lee, A.Y., Salmen, B., Law, J.W., Wotjak, C.T., Schweizer, M., Dityatev, A., and Schachner, M. (2004). Conditional ablation of the neural cell adhesion molecule reduces precision of spatial learning, long-term potentiation, and depression in the CA1 subfield of mouse hippocampus. J. Neurosci. 24, 1565-1577.

Cremer, H., Lange, R., Christoph, A., Plomann, M., Vopper, G., Roes, J., Brown, R., Baldwin, S., Kraemer, P., Scheff, S., et al. (1994). Inactivation of the N-CAM gene in mice results in size reduction of the olfactory bulb and deficits in spatial learning. Nature 367, 455-459.

Dalva, M.B., McClelland, A.C., and Kayser, M.S. (2007). Cell adhesion molecules: signalling functions at the synapse. Nat. Rev. Neurosci. 8, 206-220.
Fukuda, M., Kanno, E., Ishibashi, K., and Itoh, T. (2008). Large scale screening for novel rab effectors reveals unexpected broad Rab binding specificity. Mol. Cell. Proteomics 7, 1031-1042.

Giridharan, S.S., and Caplan, S. (2014). MICAL-family proteins: Complex regulators of the actin cytoskeleton. Antioxid. Redox Signal. 20, 2059-2073.

Glanzman, D.L., Kandel, E.R., and Schacher, S. (1990). Targetdependent structural changes accompanying long-term synaptic facilitation in Aplysia neurons. Science 249, 799-802.

Hoeffer, C.A., Sanyal, S., and Ramaswami, M. (2003). Acute induction of conserved synaptic signaling pathways in Drosophila melanogaster. J. Neurosci. 23, 6362-6372.

Koh, Y.H., Ruiz-Canada, C., Gorczyca, M., and Budnik, V. (2002). The Ras1-mitogen-activated protein kinase signal transduction pathway regulates synaptic plasticity through fasciclin II-mediated cell adhesion. J. Neurosci. 22, 2496-2504.

Kohsaka, H., Takasu, E., and Nose, A. (2007). In vivo induction of postsynaptic molecular assembly by the cell adhesion molecule Fasciclin2. J. Cell. Biol. 179, 1289-1300.

Lin, D.M., and Goodman, C.S. (1994). Ectopic and increased expression of Fasciclin II alters motoneuron growth cone guidance. Neuron 13, 507-523.

Mathew, D., Popescu, A., and Budnik, V. (2003). Drosophila amphiphysin functions during synaptic Fasciclin II membrane cycling. J. Neurosci. 23, 10710-10716.

Mayford, M., Barzilai, A., Keller, F., Schacher, S., and Kandel, E.R. (1992). Modulation of an NCAM-related adhesion molecule with long-term synaptic plasticity in Aplysia. Science 256, 638-644.

Michael, D., Martin, K.C., Seger, R., Ning, M.M., Baston, R., and Kandel, E.R. (1998). Repeated pulses of serotonin required for long-term facilitation activate mitogen-activated protein kinase in sensory neurons of Aplysia. Proc. Natl. Acad. Sci. USA 95, 18641869.

Muller, D., Wang, C., Skibo, G., Toni, N., Cremer, H., Calaora, V., Rougon, G., and Kiss, J.Z. (1996). PSA-NCAM is required for activity-induced synaptic plasticity. Neuron 17, 413-422.

Nahm, M., Kim, S., Paik, S.K., Lee, M., Lee, S., Lee, Z.H., Kim, J., Lee, D., Bae, Y.C., and Lee, S. (2010a). dCIP4 (Drosophila Cdc42-interacting protein 4) restrains synaptic growth by inhibiting the secretion of the retrograde Glass bottom boat signal. J. Neurosci. 30, 8138-8150.

Nahm, M., Long, A.A., Paik, S.K., Kim, S., Bae, Y.C., Broadie, K., and Lee, S. (2010b). The Cdc42-selective GAP rich regulates postsynaptic development and retrograde BMP transsynaptic signaling. J. Cell. Biol. 191, 661-675.

Packard, M., Mathew, D., and Budnik, V. (2003). FASt remodeling of synapses in Drosophila. Curr. Opin. Neurobiol. 13, 527-534.

Sakane, A., Honda, K., and Sakai, K. (2010). Rab13 regulates neurite outgrowth in PC12 cells through its effector protein, JRAB/MICAL-L2. Mol. Cell Biol. 30, 1077-1087.

Schuster, C.M., Davis, G.W., Fetter, R.D., and Goodman, C.S. (1996a). Genetic dissection of structural and functional components of synaptic plasticity. I. Fasciclin II controls synaptic stabilization and growth. Neuron 17, 641-654.

Schuster, C.M., Davis, G.W., Fetter, R.D., and Goodman, C.S. (1996b). Genetic dissection of structural and functional components of synaptic plasticity. II. Fasciclin II controls presynaptic structural plasticity. Neuron 17, 655-667.

Sharma, M., Giridharan, S.S., Rahajeng, J., Naslavsky, N., and Caplan, S. (2009). MICAL-L1 links EHD1 to tubular recycling endosomes and regulates receptor recycling. Mol. Biol. Cell 20, 5181-5194.

Terai, T., Nishimura, N., Kanda, I., Yasui, N., and Sasaki, T. (2006). JRAB/MICAL-L2 is a junctional Rab13-binding protein mediating the endocytic recycling of occludin. Mol. Biol. Cell 17, 2465-2475.

Verstreken, P., Ohyama, T., and Bellen, H.J. (2008). FM 1-43 labeling of synaptic vesicle pools at the Drosophila neuromuscular junction. Methods Mol. Biol. 440, 349-369.

Yamamura, R., Nishimura, N., Nakatsuji, H., Arase, S., and Sasaki, T. (2008). The interaction of JRAB/MICAL-L2 with Rab8 and Rab13 coordinates the assembly of tight junctions and adherens junctions. Mol. Biol. Cell. 19, 971-983. 